\title{
Phase transition in the binary mixture of jammed particles with large size dispersity
}

\author{
Yusuke Hara, ${ }^{1, *}$ Hideyuki Mizuno, ${ }^{1}$ and Atsushi Ikeda ${ }^{1,2}$ \\ ${ }^{1}$ Graduate School of Arts and Science, University of Tokyo, Komaba, Tokyo 153-8902, Japan \\ ${ }^{2}$ Research Center for Complex Systems Biology, Universal Biology Institute, University of Tokyo, Komaba, Tokyo 153-8902, Japan
}

(Received 22 September 2020; accepted 20 April 2021; published 3 May 2021; corrected 6 May 2021)

\begin{abstract}
It has been well established that particulate systems show the jamming transition and critical scaling behaviors associated with it. However, our knowledge is limited to (nearly) monodisperse systems. Recently, a binary mixture of jammed particles with large size dispersity was studied, and it was suggested that two distinct jammed phases appeared. Here, we conduct a thorough numerical study on this system with a special focus on the statistics of and finite-size effects on the fraction of small particles that participate in the rigid network. We present strong evidence that two distinct jammed phases appear depending on the pressure and composition of two species, which are separated by the first-order phase transition. In one of two phases, only large particles are jammed, whereas both small and large particles are jammed in the other phase. Interestingly, the finite size scaling shows the transition is similar to the first-order transition in the random-field Ising model. We also describe the phase diagram in the pressure-composition plane, where the first-order phase transition line terminates at a critical point. In addition, we investigate the mechanical properties in terms of the elastic moduli over the phase diagram and find that discontinuous changes in elastic moduli emerge across the phase transition. Finally, we show the scaling laws of the elastic moduli in each jammed phase are not in consistent with those in the monodisperse systems.
\end{abstract}

DOI: 10.1103/PhysRevResearch.3.023091

\section{INTRODUCTION}

Jammed particulate systems are ubiquitous in our lives. Emulsions, colloidal suspensions, and granular materials are examples of jammed systems, where constituent particles are randomly jammed [1]. The random structure of jammed systems is one source of difficulties in understanding these systems.

One of the simplest models for jammed particulate systems is the assemblies of athermal particles that interact via shortrange repulsive potentials. When we compress these particles from the low density, the system gains rigidity at the density called the jamming point. This phenomenon is known as the jamming transition established by many previous works, e.g., Ref. [2]. The geometrical, mechanical, and vibrational properties of the system are known to follow the critical power law near the transition where the distance from the jamming points plays the role of a control parameter [2-16]. Recently, jammed systems composed of dimer-shaped particles were shown to exhibit the same critical laws as a sphere packings [17-19]. In the last two decades, numerical and theoretical studies of these systems have developed to a large extent, and we have a

\footnotetext{
*hara-yusuke729@g.ecc.u-tokyo.ac.jp

Published by the American Physical Society under the terms of the Creative Commons Attribution 4.0 International license. Further distribution of this work must maintain attribution to the author(s) and the published article's title, journal citation, and DOI.
}

good level of understanding of the critical behaviors near the jamming transition in nearly monodisperse jammed particles.

However, the jammed particles in reality are not monodisperse and are composed of particles of multiple sizes [20-22]. In particular, when the sizes of larger and smaller particles are quite different, these systems can exhibit phenomena that are never observed in the monodisperse systems [23,24]. For example, mixtures of large colloidal particles and small polymers are known to show rich phenomena, including the emergence of two distinctive colloidal glass phases and a peculiar nonlinear mechanical response [25-28].

Despite its theoretical and practical importance, the impacts of the polydispersity with a large size ratio on the characteristic features of jammed packings are poorly understood. The simplest system to study the effects of polydispersity can be a binary mixture of particles with large size dispersity. The packings of binary mixtures have been studied to improve the packing efficiency. It is now well known that an injection of fine particles to coarse large structures realizes the random close packing with a higher packing fraction than monodispersity [29-35].

In addition, the nature of the jamming transition in binary systems was studied. Xu et al. showed that binary mixtures of equal numbers of small and large particles did not change the critical behavior near the jamming transition even if their sizes were significantly different [36]. Kumar et al. studied the effects on the bulk moduli by injecting a few percent of fine particles into a volume of large particles [37].

More recently, it was suggested that binary mixtures of large and small particles have two different types of jammed phases, i.e., the jammed state of only large particles and that of 
both large and small particles. Prasad et al. [34] studied binary systems at zero pressure $P=0$ for various particle size ratios and composition ratios. They focused on the fraction of rattler particles as the order parameter and the first derivative of the order parameter with respect to the composition ratio, which is called susceptibility. They showed that the susceptibility increases with the system size at the transition point, which establishes the presence of the phase transition between the two phases. Petit et al. [38] studied binary systems at finite pressure $P>0$. They calculated the order parameter and the susceptibility, which is similar to that defined in Ref. [34], and observed the sign that the transition line extends into the jammed region $P>0$, and terminates at some critical density, although the system-size dependence of the susceptibility was not investigated. Note that the presence of two different types of jammed states was also reported for binary mixtures of two-dimensional disks [33]. In the present paper, we investigate the structural and mechanical properties of the packing of binary mixtures of particles with large size dispersity. The size ratio is fixed to be 6 , and we study the packings at finite pressure $P>0$. First, we thoroughly study the transition between two jammed phases. We focus on the fraction of small particles that contribute to the rigidity of the system as the order parameter. Unlike the previous works, we focus on the sample variance of the order parameter, which is also called the susceptibility. We quantitatively show that this susceptibility becomes proportional to the system size at the transition, which is the hallmark of the first-order phase transition. Moreover, we analyzed the susceptibility introduced in Ref. [34] and found that it becomes proportional to the square root of the system size, suggesting that the first-order transition in the model is similar to that in the random-field Ising model. By analyzing the susceptibility in various state points, we locate the first-order transition line that terminates at the critical point. Second, we analyzed the mechanical properties of the system. We show that the bulk and shear moduli exhibit a discontinuous change at the first-order transition. We also show that the shear modulus exhibits critical behavior near the jamming threshold in both phases, similar to the monodisperse system.

This paper is organized as follows. In Sec. II, we introduce the detailed description of the system in interest. In Sec. III, we present our simulation results. In Sec. III A, we focus on the analysis of the structural characterization at the fixed pressure and establish the first-order transition. Then, in Sec. III B, we extend the analysis to a broad range of pressure and reveal the phase diagram. Finally, in Sec. III C, the mechanical properties such as the bulk and shear moduli are studied. We provide the summary and conclusion in Sec. IV.

\section{NUMERICAL METHOD}

\section{A. System description}

We study the jammed packings composed of the binary mixtures of large and small particles in three spatial dimensions. The diameter of the large particle is 6 times that of the small particles. We denote the volumes of large and small particles as $v_{l}$ and $v_{s}$, respectively, where $v_{l}=6^{3} v_{s}$. The numbers of large and small particles are denoted by $N_{L}$ and $N_{S}$, respectively. These particles interact via a finite-range, purely repulsive potential [2]; two particles interact with each other only when they are in contact. The interaction potential is the following harmonic potential

$$
v_{i j}\left(r_{i j}\right)=\frac{\epsilon}{2}\left(r_{i j}-\sigma_{i j}\right)^{2} \Theta\left(1-\frac{r_{i j}}{\sigma_{i j}}\right),
$$

where $\sigma_{i j}$ is the sum of the radii of particles $i$ and $j, r_{i j}$ is the distance between the centers of particles $i$ and $j$, and $\Theta(x)$ is Heaviside step function; $\Theta(x)=1$ for $x>0$, otherwise $\Theta(x)=0$. We set the unit of the length and the energy as the diameter of the small particle and $\epsilon$, respectively.

The state point of this system can be characterized by two relevant parameters. The first one is the volume ratio of small particles:

$$
X_{S}=\frac{N_{S} v_{s}}{N_{S} v_{s}+N_{L} v_{l}} .
$$

In this paper, we focus on the range of $0.05 \leqslant X_{S} \leqslant 0.25$, where the number of small particles is approximately 10 to 70 times that of large particles. The second is the pressure:

$$
P=-\sum_{\langle i j\rangle} r_{i j} \frac{d v_{i j}\left(r_{i j}\right)}{d r_{i j}},
$$

where $\sum_{\langle i j\rangle}$ is the summation over all contacting pairs of particles. The packing fraction is sometimes used as the control parameter, but we use the pressure because it enables us to more properly study the critical behaviors. Packings with desired $\left(P, X_{S}\right)$ are obtained by iterations of compression and decompression of the system, as described below.

There are three possible quantities to define the system size: $N_{S}+N_{L}, N_{S}$, and $N_{L}$. Although previous studies [34,38] of binary mixtures use the total number of particles $N_{S}+N_{L}$ to express the system size, we can also use the number of small particles $N_{S}$ or large particles $N_{L}$. In this work, we use $N_{S}$ to precisely study the size effect on the susceptibility because $N_{S}$ explicitly appears in the definition of the susceptibility (see Sec. III A). On the other hand, we use $N_{L}$ to discuss the phase diagram because this choice enables us to explore the broad range of $X_{S}$ (see Sec. III B).

\section{B. System preparation}

We generate the jammed packing of desired pressures using the iterative compression and decompression of the system and the FIRE algorithm to minimize the energy [39]. The condition to terminate the relaxation algorithm is $\max _{i, \alpha}\left(F_{i}^{\alpha}\right) \leqslant$ $10^{-13}$, where $\alpha$ is the spatial index, and $i$ is the index of the particles.

We prepare the configuration below the jamming point by relaxing the random configuration with a low packing fraction to the mechanical equilibrium. Then, we compress the system with fixed increment $\Delta \phi_{\text {ini }}=0.01$, and the systems are relaxed to mechanical equilibrium in each step. We continue to increase the packing fraction until the pressure of the equilibrated configuration exceeds the target pressures. The procedures are terminated if the pressure of the configuration is consistent with the target pressures within the acceptable error; in this case, $\delta P=\left|P-P_{\text {tag }}\right| \leqslant 10^{-2} P_{\text {tag. }}$. When this condition is not satisfied, we (1) decompress with the rate 
$\Delta \phi$ and (2) compress with the new rate $\Delta \phi_{\text {new }}=0.5 \Delta \phi$. The iterative use of the procedures brings the system to the desired pressure.

We generate at least 100 packings at each state point. We denote the average of physical quantities $x$ in the ensemble of the packings generated at a state point by $\langle x\rangle_{\text {sample }}$. We also analyze the statistics of quantities $x$ in the ensemble and calculate the probability distribution $P(x)$. Note that the ordering of large particles occurs in some packings. We detect these packings by calculating the local bondorder parameter of the structure of large particles [40]. These packings are rare, and we exclude them from our statistical ensemble.

\section{RESULTS}

\section{A. Transition between two jammed phases}

In this section, we study the structural properties of the packings with changing $X_{S}$ at the fixed pressure $P=10^{-3}$. We will show that the system exhibits two distinct jammed phases, which are separated by a first-order phase transition. One phase is named the L phase, where only large particles are jammed, and the other is the LS phase, where both large and small particles are jammed. To characterize these phases, we focus on the fraction of small particles that participate in the connected network of particles,

$$
R_{S}=\frac{\left(N_{S}-N_{S}^{r}\right)}{N_{S}} .
$$

Here, $N_{S}^{r}$ is the number of small particles that are rattlers, which are defined as the particles whose contact number is less than $d+1=4$ (where $d=3$ is a spatial dimension). Identification of rattlers are done by the iterative procedures of counting the contact number of individual particles $Z_{i}$, and removal of particles with $Z_{i}<d+1$ from the packings. We stop this iteration when no more particles with $Z_{i}<d+1$ exist in the packing. Note that the FIRE algorithm produces the packings in which the most of rattlers are with $Z_{i}=0$, and the iteration to find rattlers ends in a few cycles. We prepare at least 200 packings at each state point $\left(P=10^{-3}, X_{S}\right)$ and calculate $R_{S}$ for each packing.

First, we focus on $\left\langle R_{S}\right\rangle_{\text {sample }}$, which is the mean value of $R_{S}$ in the ensemble of at least 200 packings at each state point. Figure 1 shows $\left\langle R_{S}\right\rangle_{\text {sample }}$ vs $X_{S}$ for various system sizes $N_{S}$. $\left\langle R_{S}\right\rangle_{\text {sample }}$ monotonically increases with $X_{s}$; namely, a larger fraction of small particles corresponds to more small particles participating in the connected network. Notably, the change in $\left\langle R_{S}\right\rangle_{\text {sample }}$ becomes steeper when the system size increases. This implies a phase transition between the states with $R_{S} \approx 0$ and $R_{S} \approx 1$ in the thermodynamic limit, and $R_{S}$ is an order parameter in this phase transition.

To clarify whether this is a genuine phase transition, we analyze the statistics of $R_{S}$. First, Fig. 2 shows the probability distribution of the order parameter $R_{S}$ over 400 different packings for several state points. When $X_{S}=0.196$, the distribution has a single peak at $R_{S} \approx 0$; most small particles are rattlers. When $X_{S}=0.209$, the distribution also has a single peak, but it is located at $R_{S} \approx 1$; most small particles participate in the connected network of particles. At $X_{S}=0.202$, the distribution shows the double peaks at $R_{S} \approx 0$ and 1 ,

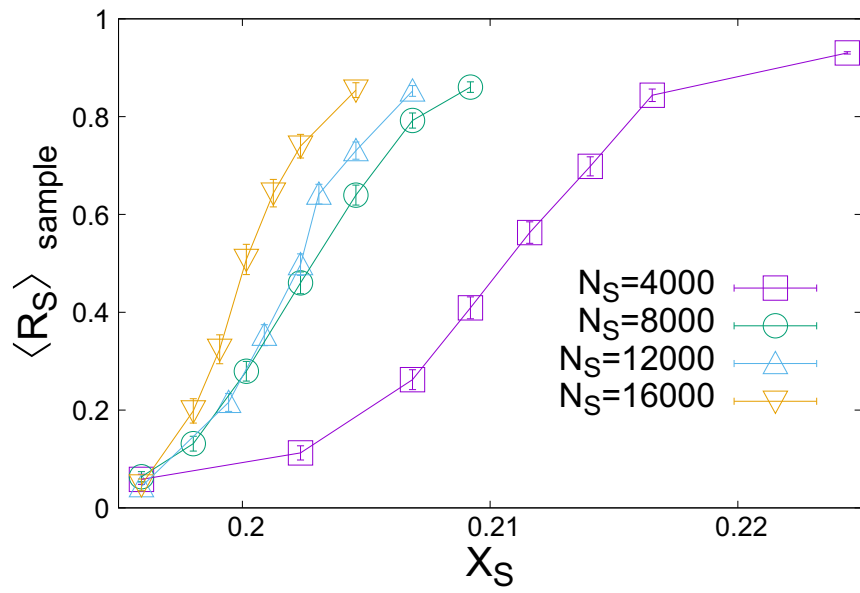

FIG. 1. Mean value of the order parameter $\left\langle R_{S}\right\rangle_{\text {sample }}$ vs $X_{S}$ at $P=10^{-3}$. Different symbols correspond to system sizes $N_{S}=$ $4000,8000,1200,16000$. For $N_{S}=4000$, the plotted interval of $X_{S}$ corresponds with $N_{L} \in[64,76]$. For $N_{S}=8000$, the plotted interval of $X_{S}$ corresponds with $N_{L} \in[140,152]$. For $N_{S}=12000$, the plotted interval of $X_{S}$ corresponds with $N_{L} \in[213,228]$. For $N_{S}=$ 16000 , the plotted interval of $X_{S}$ corresponds with $N_{L} \in[288,304]$. The number of samples is 400 for $N_{S}=4000,8000,12000$ and 200 for $N_{S}=16000$, respectively. The increase in $\left\langle R_{S}\right\rangle_{\text {sample }}$ becomes steeper when the system size increases.

respectively; most small particles are rattlers in some packings, while most small particles participate in the connected network of particles in the other packings. The appearance of the bimodal distribution strongly suggests that this is the first-order phase transition between two phases.

Next, we consider the system-size dependence of this behavior. For this purpose, we calculate the sample variance of $N_{S}-N_{S}^{r}$, which is an extensive form of the order parameter $R_{S}$,

$$
\begin{aligned}
& \left\langle\left(N_{S}-N_{S}^{r}\right)^{2}\right\rangle_{\text {sample }}-\left\langle N_{S}-N_{S}^{r}\right\rangle_{\text {sample }}^{2} \\
& =N_{S}^{2}\left(\left\langle R_{S}^{2}\right\rangle_{\text {sample }}-\left\langle R_{S}\right\rangle_{\text {sample }}^{2}\right) .
\end{aligned}
$$

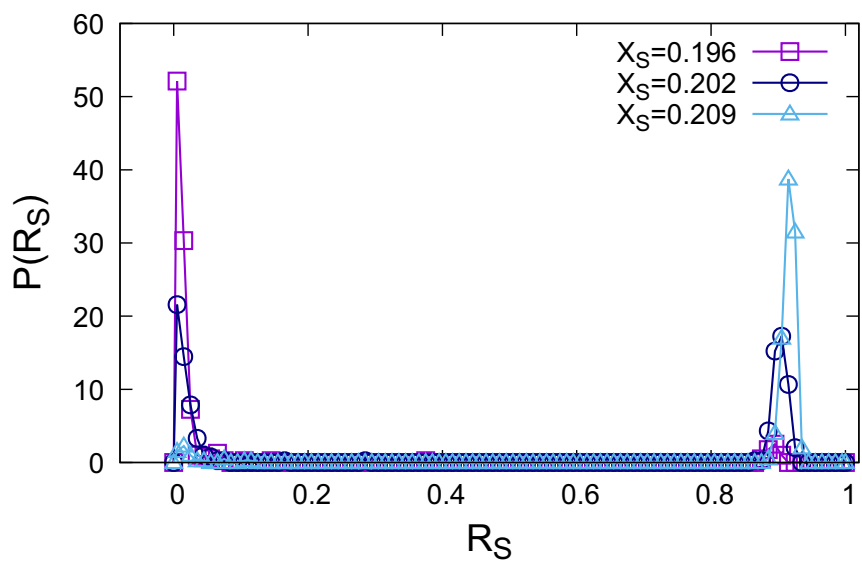

FIG. 2. Probability distributions of order parameter $R_{S}$ over 400 independent packings at $P=10^{-3}$ for $N_{S}=8000$. The distribution becomes bimodal at $X_{S}=0.202$. 

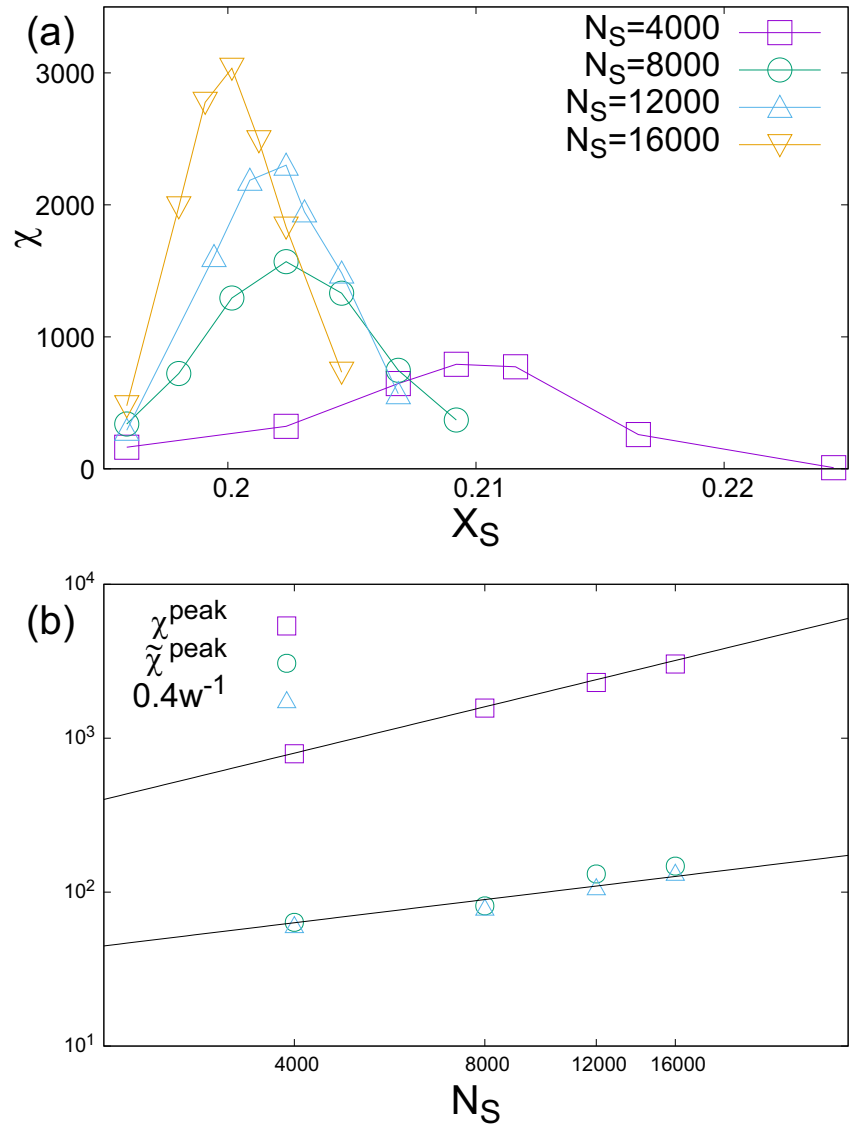

FIG. 3. (a) Susceptibility (variance of the order parameters) $\chi$ vs $X_{S}$ at various system sizes $N_{S}$. The susceptibility exhibits the strong finite size effects. (b) Peak height of the susceptibilities $\chi^{\text {peak }}$ and $\tilde{\chi}^{\text {peak }}$. The dotted and solid lines indicate the linear and the square root dependence on system size $N_{S}$, respectively. The inverses of the widths of the transition region $0.4 / w$ are also plotted. The pressure is $P=10^{-3}$.

By dividing this variance by the system size $N_{S}$, we introduce the susceptibility as

$$
\chi=N_{S}\left(\left\langle R_{S}^{2}\right\rangle_{\text {sample }}-\left\langle R_{S}\right\rangle_{\text {sample }}^{2}\right) .
$$

Figure 3(a) shows $\chi$ vs $X_{S}$. Clearly, $\chi$ exhibits a peak, whose height increases with the system size. When the transition is of first-order nature, the peak height is expected to be proportional to the system size. To study this point, Fig. 3(b) shows the system-size dependence of the peak height of the susceptibility, which is denoted by $\chi^{\text {peak }}$. The data are consistent with $\chi^{\text {peak }} \propto N_{S}$, which confirms that the transition between two phases is first order.

One can consider another susceptibility,

$$
\tilde{\chi}=\frac{d}{d X_{S}}\left\langle R_{S}\right\rangle_{\text {sample }},
$$

which was introduced in Ref. [34]. When the average is over the thermal fluctuations, these two types of susceptibilities, $\chi$ and $\tilde{\chi}$, should be equivalent due to the fluctuation formula. However, this is not necessarily the case when the randomness does not originate from thermal fluctuation. Indeed, at the first-order transition in the random-field Ising model, the

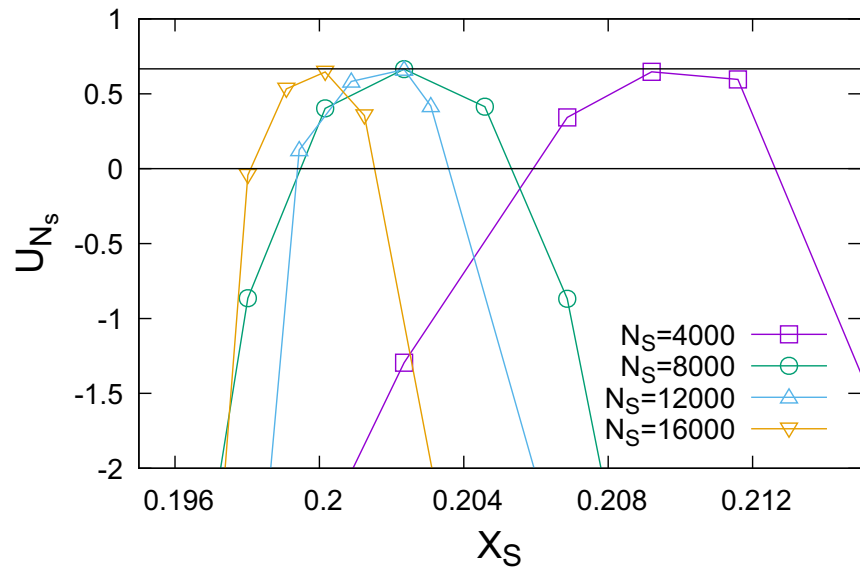

FIG. 4. Binder parameter $U_{N_{S}}$ vs $X_{S}$. The horizontal dashed line indicates $2 / 3$.

disconnected susceptibility (similar to $\chi$ ) is proportional to the system size, while the connected susceptibility (similar to $\tilde{\chi})$ is to the square-root of the system size $[41,42]$. Previously, Ref. [34] showed that $\tilde{\chi}$ increases with the system size but did not determine its functional form. Here, we calculate $\tilde{\chi}$ using our data sets by performing the numerical differentiation of the data presented in Fig. 1 [43]. We find that $\tilde{\chi}$ has a peak, and the peak height increases with the system size, as is consistent with Ref. [34]. In Fig. 3(b), we plot the system-size dependence of the peak height of $\tilde{\chi}$, which is denoted by $\tilde{\chi}^{\text {peak }}$. The system-size dependence of $\tilde{\chi}^{\text {peak }}$ is weaker than $\chi^{\text {peak }}$ and consistent with $\tilde{\chi}^{\text {peak }} \propto \sqrt{N_{S}}$. This suggests that the first-order transition in this model is different from that in the pure Ising model but similar to that in the random-field Ising model. Note that we also analyzed the data sets presented in Ref. [34] and found that they are consistent with the square-root dependence on the system size. Additionally, we analyze the width of the transition region defined by $w=X_{S}\left(\left\langle R_{S}\right\rangle_{\text {sample }}=0.7\right)-X_{S}\left(\left\langle R_{S}\right\rangle_{\text {sample }}=0.3\right)$ [44]. We plot $0.4 / w$ in Fig. 3(b), where the factor of 0.4 is included to directly compare with $\tilde{\chi}$. This figure clearly shows $w \propto 1 / \sqrt{N_{S}}$, which is expected for the first-order transition in the random-field Ising model [41]. Therefore, our results suggest that the present model exhibits a first-order transition similar in nature to that in the random-field Ising model. Note that similar behavior was reported for the yielding transition in amorphous solids [42].

Finally, we evaluate the reduced cumulant of the order parameter defined by

$$
U_{N_{S}}=1-\frac{\left\langle\left(R_{S}-\left\langle R_{S}\right\rangle_{\text {sample }}\right)^{4}\right\rangle_{\text {sample }}}{3\left(\left\langle\left(R_{S}-\left\langle R_{S}\right\rangle_{\text {sample }}\right)^{2}\right\rangle_{\text {sample }}\right)^{2}},
$$

which is known as the binder parameter [45]. Figure 4 shows $U_{N_{S}}$ vs $X_{S}$ at various system sizes. Regardless of the system size, the binder parameter converges to $\frac{2}{3}$, which indicates the appearance of the bimodal distribution and a common feature of the first-order transition. This plot also shows the negative dip of the binder parameter, which is another common feature of first-order transitions. 


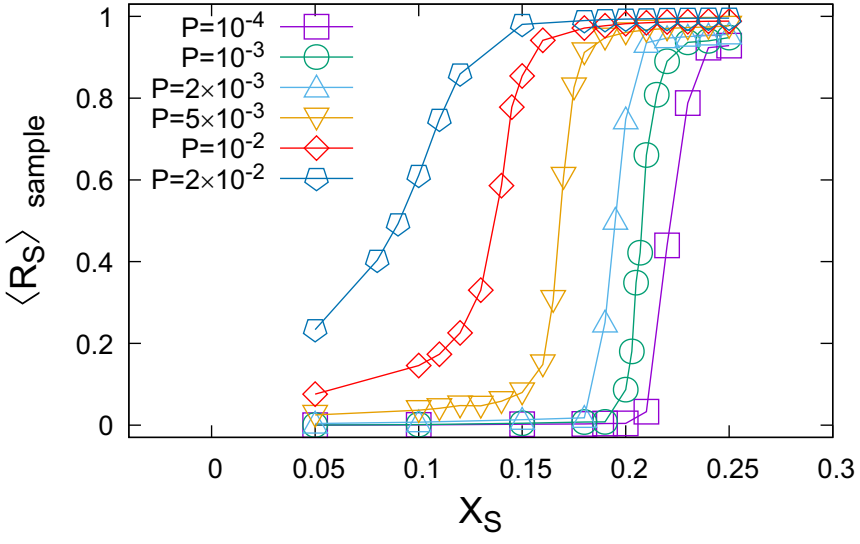

FIG. 5. Mean value of order parameter $\left\langle R_{S}\right\rangle_{\text {sample }}$ vs $X_{S}$ at various pressures for $N_{L}=64$.

In summary, we provided strong evidence for the first-order transition in the jammed phase, which is the transition between the jammed phase with only large components jamming (L phase) and that with both components jamming (LS phase).

\section{B. Phase diagram}

In the previous section, we established the presence of the first-order transition at $P=10^{-3}$, where fraction $R_{S}$ of small particles participating in the connected network is the order parameter. In this section, we extend the analysis to the broad range of pressures and determine the phase diagram of the packing of a binary mixture of particles. As a consequence, we will show that the first-order transition line terminates at a finite pressure.

To explore the region of small $X_{S}$, here, we choose to fix $N_{L}$. This is because it is numerically difficult to explore such regions with fixing $N_{S}$. Specifically, when we explore the phase space at $X_{S} \rightarrow 0$ while fixing $N_{S}, N_{L}$ diverges. The only slight concern is that there is a nontrivial variation of $N_{S}$ when comparing the peak heights of different $N_{L}$. We check that such a variation at $P=10^{-3}$ is small, and it is expected that the choice of the system size does not qualitatively change the conclusion of the following results.

Figure 5 plots $\left\langle R_{S}\right\rangle_{\text {sample }}$ vs $X_{S}$ at various pressure. At low pressure, $R_{S}$ exhibits discontinuous jumps around $X_{S} \approx 0.2$, which suggests the first-order transition as discussed in the previous section. However, at high pressure, e.g., $P=2 \times$ $10^{-2}$, the increase becomes much milder. At this pressure, $R_{S}$ appears to smoothly change along $X_{S}$, which implies the disappearance of the first-order transition.

To confirm this point in more detail, we analyze the statistics of $R_{S}$ at high pressure as performed at low pressure in the previous section. Figure 6 shows the distribution of order parameter $R_{s}$ over different packings at $P=2 \times 10^{-2}$. The evolution of the distribution with $X_{S}$ is completely different from that at $P=10^{-3}$. The distribution always has only a single peak at all $X_{S}$, and the peak only continuously shifts with $X_{S}$. In addition, Fig. 7 plots $\chi$ vs $X_{S}$ at the same pressure. Clearly, $\chi$ does not depend on the system size, which is a totally different observation in Fig. 3. These distinctive behaviors provide strong evidence for the lack of the first-order

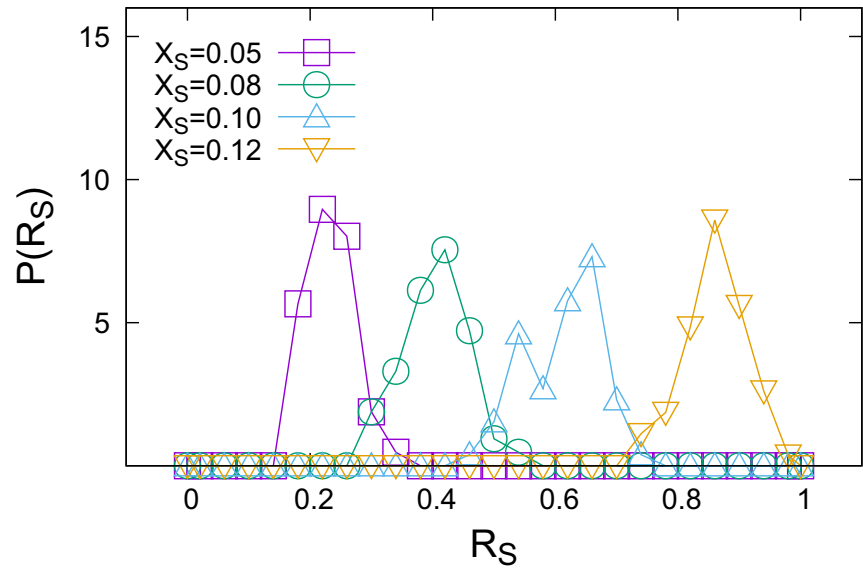

FIG. 6. Probability distributions of order parameter $R_{S}$ at $P=$ $2 \times 10^{-2}$ for $N_{L}=128$. The distribution has only a single peak, and the peak continuously moves with $X_{S}$.

transition at higher pressure $P=2 \times 10^{-2}$. Thus, there is a critical point at a finite pressure $P_{c}$ between $P=10^{-3}$ and $P=2 \times 10^{-2}$.

We repeated the above analysis at different pressures, plotted $\chi$ vs $X_{S}$, and observed the peak. At pressure $P$, we denote the peak height by $\chi^{\text {peak }}(P)$ and its position in $X_{S}$ axis by $X_{S}^{\text {peak }}(P)$. In Fig. 8, $\chi^{\text {peak }}(P)$ vs $P$ is plotted for various system sizes. $\chi^{\text {peak }}(P)$ depends on the system size at low pressure and not at high pressure. We determine that the first-order transition occurs at pressure $P$ if $\chi^{\text {peak }}(P)$ exhibits the strong system-size dependence even in our largest system size. Then, the first-order transition point is estimated as $\left(P, X_{S}^{\text {peak }}(P)\right)$, which are shown as open symbols in Fig. 9. We also observe that $\chi^{\text {peak }}(P)$ increases with decreasing $P$ in the high-pressure regime, which suggests that $\chi^{\text {peak }}$ diverges at critical pressure $P_{c}$. To precisely determine $P_{c}$, we must simulate much larger

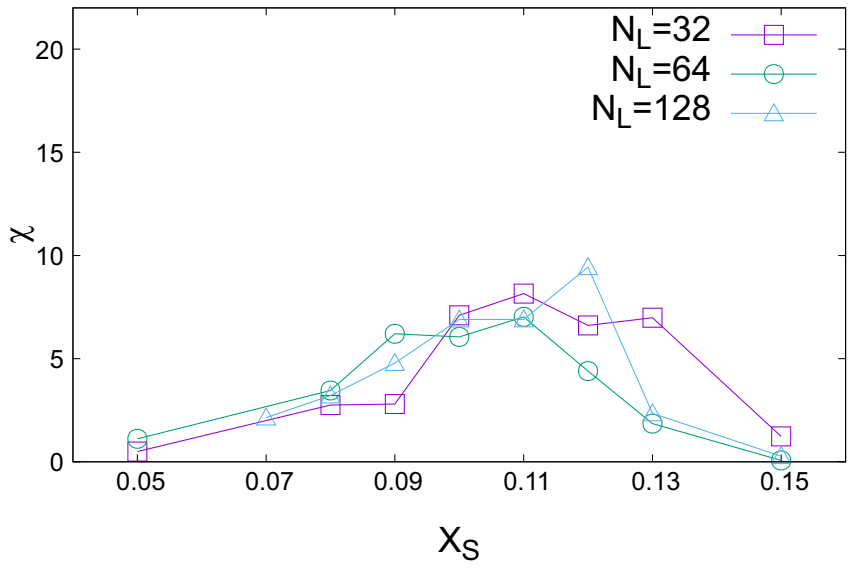

FIG. 7. Susceptibility (variance of the order parameters) $\chi$ vs $X_{S}$ at various system sizes $N_{L}$. The pressure is $P=2 \times 10^{-2}$. For $N_{L}=32$, the plotted interval of $X_{S}$ corresponds with $N_{S} \in$ [364, 1220]. For $N_{L}=64$, the plotted interval of $X_{S}$ corresponds with $N_{S} \in[728,2440]$. For $N_{L}=128$, the plotted interval of $X_{S}$ corresponds with $N_{S} \in[1456,4880]$. The susceptibility does not show the system-size dependence. 


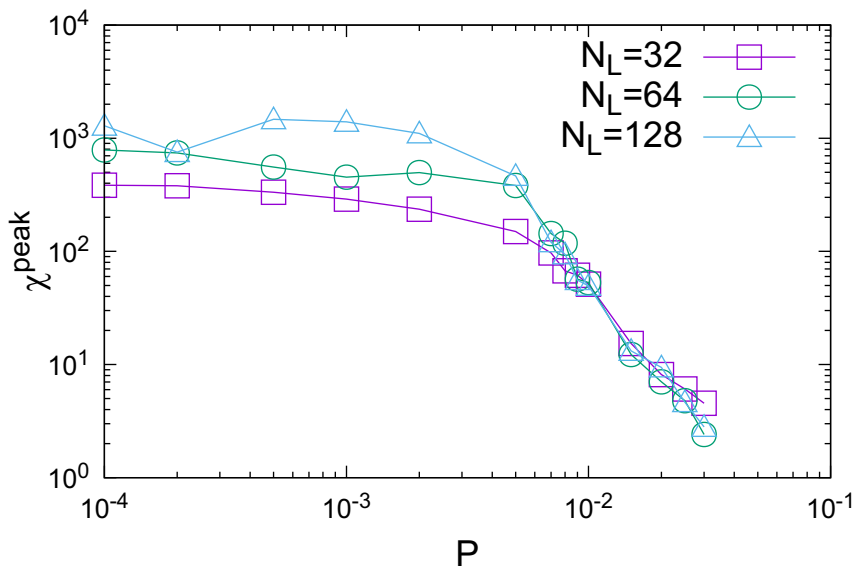

FIG. 8. The peak height of susceptibility $\chi^{\text {peak }}$ is plotted as a function of the pressure. The strong system-size dependence appears at approximately $P=2 \times 10^{-3}$.

systems, which is beyond the scope of this paper and can be addressed in the future. Here, we provide a rough estimate of $P_{c}$ as the highest pressure at which we observe the strong size effects in $\chi^{\text {peak }}: P_{c} \approx 2 \times 10^{-3}$, which is shown as a closed symbol in Fig. 9. In this phase diagram, the LS phase indicates the state with $R_{S} \approx 1$, where both components are jammed, and the $\mathrm{L}$ phase is the state with $R_{S} \approx 0$, where only the large component is jammed. At jamming point $P \rightarrow 0$, the first-order transition is located at $X_{S}=0.22$, and the transition line continues for a finite pressure. This transition line is terminated at the critical point, which is estimated to be at $X_{S}=0.195$ and $P=2 \times 10^{-3}$.

Finally, we compare the location of the transition line in our works with the results in previous works. From our data, we estimated the location of the first-order transition point to be $\left(X_{S}=0.22,\langle\phi\rangle_{\text {sample }}=0.803\right)$ for $P=10^{-4}$, where $\langle\phi\rangle_{\text {sample }}$ is the averaged packing fraction at fixed pressure. This is not far from $\left(X_{S} \approx 0.2, \phi \approx 0.8\right)$, which is the transition point at $P=0$ reported in Ref. [34] for the same size ratio. Furthermore, this is not far from $\left(X_{S}=0.21, \phi=0.81\right)$ for the size ratio of 6.66 reported in Ref. [38].

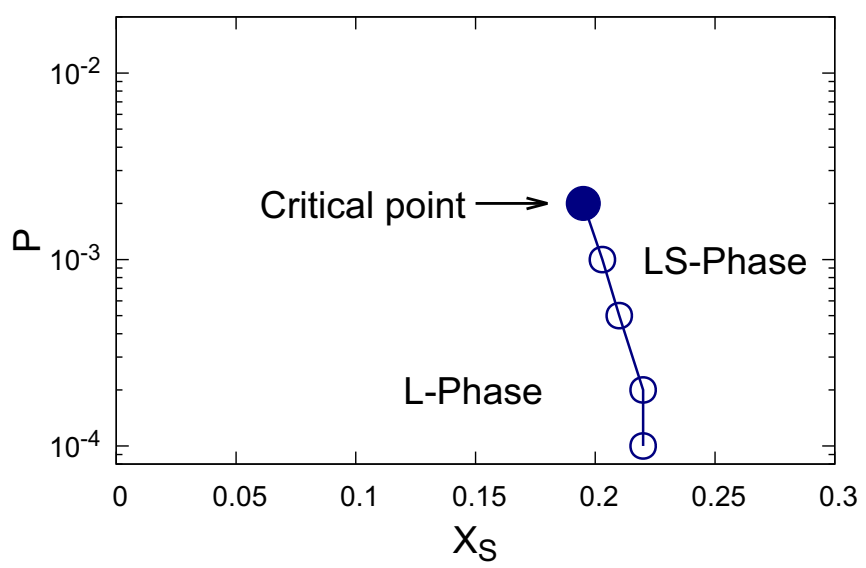

FIG. 9. Phase diagram of the packings of binary mixtures of particles.
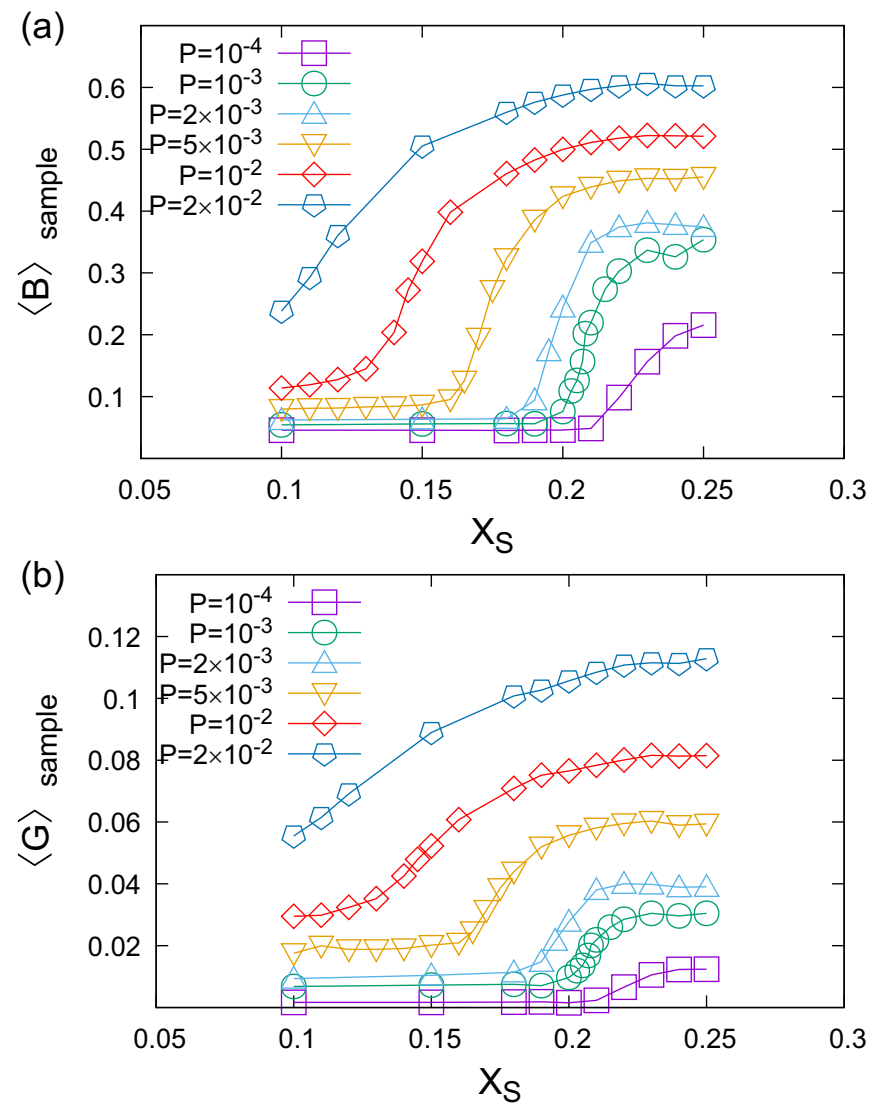

FIG. 10. (a) Mean value of bulk modulus $B$ vs $X_{S}$. The system size is fixed at $N_{L}=64$. Each symbol represents the value at a different pressure. (b) Same as (a) but for shear modulus $G$.

\section{Mechanical properties}

So far, we have established the phase diagram of the binary mixtures where there is the first-order transition line, and it terminates at the critical pressure. In this section, we explore the impact of this phase behavior on the mechanical properties of the system. We investigate bulk modulus $B$ and shear modulus $G$ of the system. Both moduli are calculated from the linear response formalism. Details of this calculation and formulation are provided in Appendix. We present the results for $N_{L}=64$ in this section.

Figure 10 shows the values of the bulk and shear moduli averaged over different packing samples. Both moduli increase when $X_{S}$ increases. This result is consistent with the behaviors of $\left\langle R_{S}\right\rangle_{\text {sample }}$ in Fig. 5; the small particles participate in the rigid network and contribute to the rigidity of the system when $X_{S}$ increases. As in the case of $\left\langle R_{S}\right\rangle_{\text {sample }}$, both moduli more dramatically increase when the pressure is decreased.

Similar to the analysis in the previous sections, we calculate the probability distributions of the bulk and shear moduli over different packings samples. Figures 11 and 12 show the behaviors of the distributions of both moduli at high and low pressures. At $P=10^{-3}$, the distributions of both moduli are bimodal as in the case of $R_{S}$. The peak at smaller moduli corresponds to the $\mathrm{L}$ phase, and the other corresponds to the LS phase. Thus, the elastic moduli also show discontinuous changes near the first-order transition in the jammed phase. 

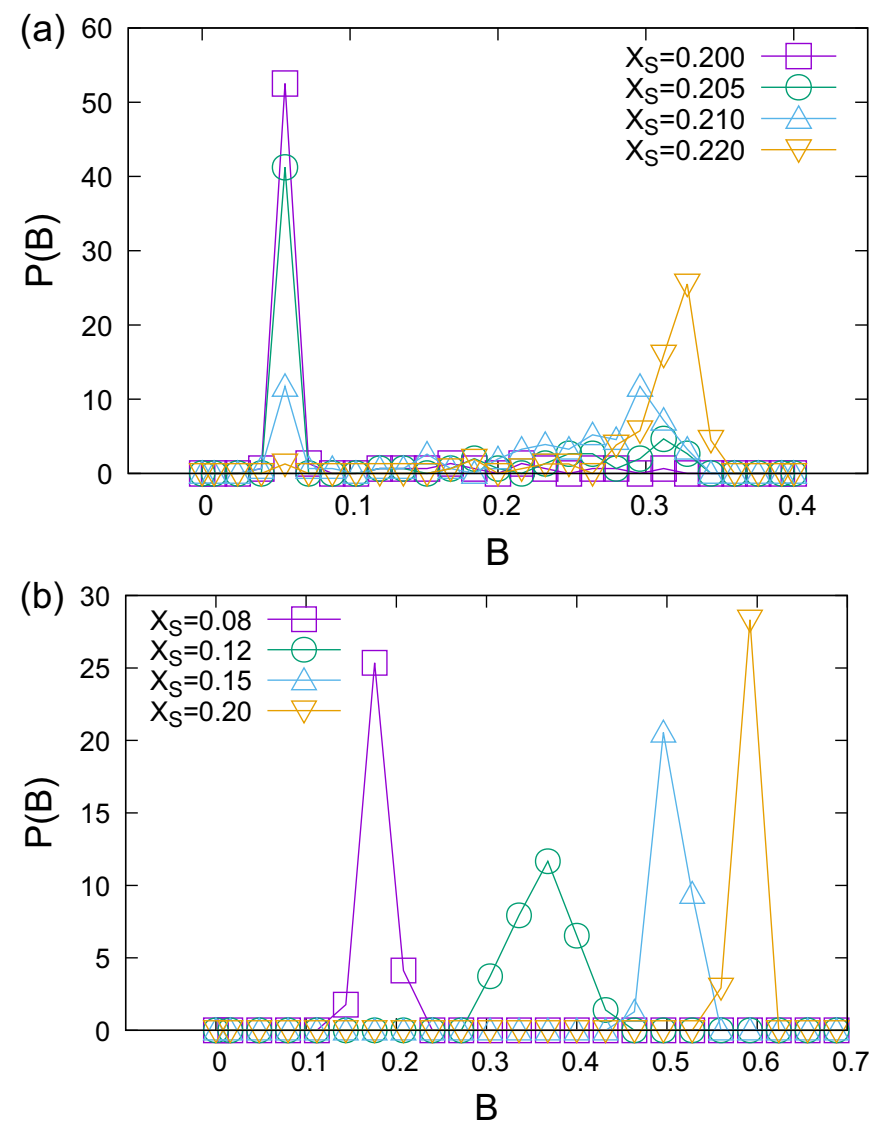

FIG. 11. Distribution of bulk moduli $B$. At the pressure of the first-order transition, the bimodal distributions are observed. (a) Distribution of $B$ at $P=10^{-3}$. (b) Distribution of $B$ at $P=2 \times 10^{-2}$.

Compare to the bulk moduli, the shear moduli have a broader distribution presumably because the fluctuation of the shear moduli becomes huge near jamming point $P \rightarrow 0$; the bimodal distributions in shear moduli are smeared by the critical fluctuations from the jamming critical phenomena. At a higher pressure than the critical pressure, the bimodal distributions of both moduli are no longer observed. Therefore, the firstorder phase transition is also characterized by the transition in mechanical properties.

Finally, we discuss the pressure dependence of the moduli. In the monodisperse systems, many physical quantities are known to follow the critical power law near the jamming transition. It has been established that the bulk modulus is independent of the pressure of the system, while shear modulus depends on the square roots of the pressure. However, it is not clear whether this jamming scaling is valid in the binary mixtures because of the discussed discontinuities of elastic moduli. In Fig. 13, we plot the average bulk modulus $\langle B\rangle_{\text {sample }}$

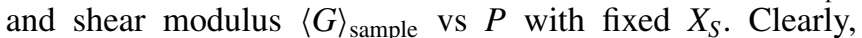
there are two branches in the plot: one branch is associated with small $X_{S}$, and the other is associated with large $X_{S}$. At intermediate $X_{S}$, we observe the jump from one branch to the other. In the lower branch, the systems are in the $\mathrm{L}$ phase, and the upper branch corresponds to the LS phase. For both branches, the jamming scalings for the monodisperse system, $B \propto P^{0}$ and $G \propto P^{1 / 2}$, are not inconsistent with our data. For the $\mathrm{L}$ phase, we find that $B$ and $G$ at various $X_{S}$ values collapse
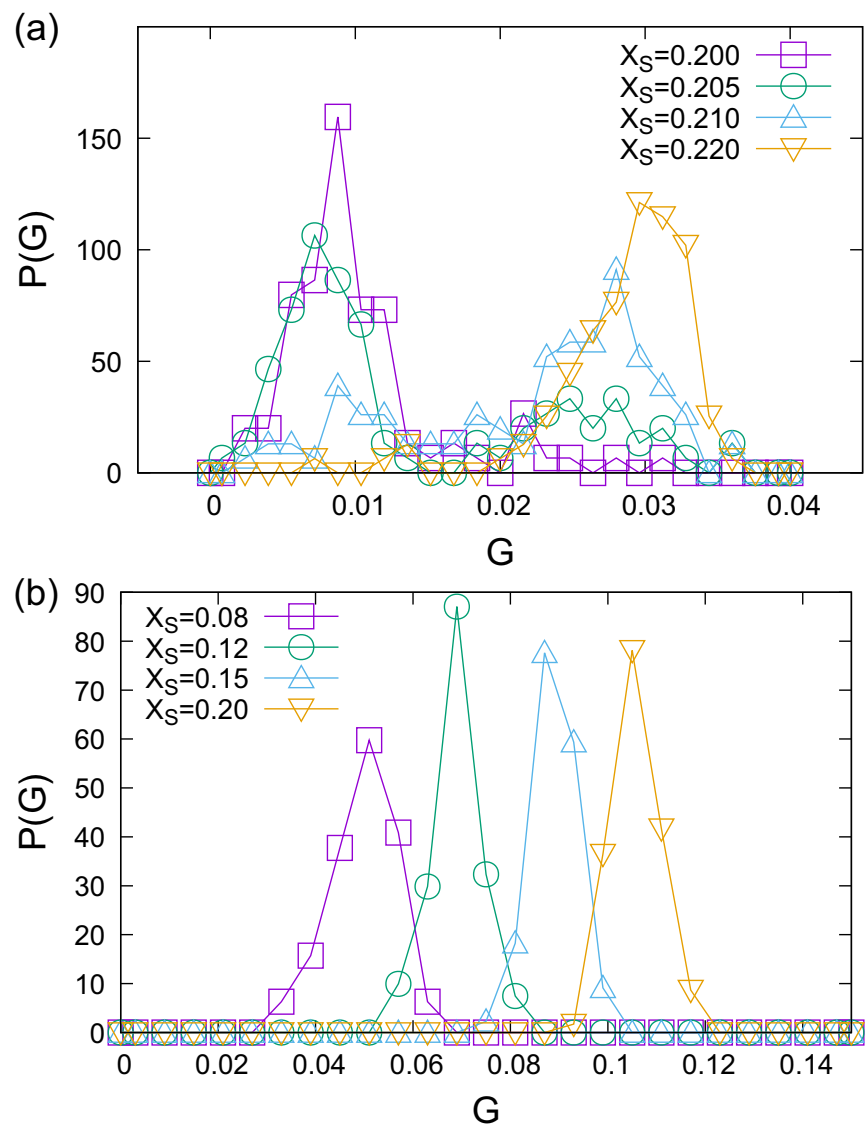

FIG. 12. Distribution of shear moduli $G$. The bimodal distributions of the shear moduli become less apparent because of the fluctuation from the jamming transition. (a) Distribution of $G$ at $P=10^{-3}$. (b) Distribution of $G$ at $P=2 \times 10^{-2}$.

well with those at $X_{S}=0$, meaning that the elastic moduli in the $\mathrm{L}$ phase are almost the same as those for the monodisperse packings of the large sphere. However, note that for the LS phase, such direct comparisons with monodisperse packings of small particles $\left(X_{S}=1\right)$ are not useful because there are still many large particles even at the largest $X_{S}$ in our work $\left(X_{S}=0.25\right)$. To discuss the data at intermediate $X_{S}$ where the transition takes place, we performed the following analysis. We classify the packings at $X_{S}=0.2$ into the $\mathrm{L}$ phase and LS phase according to the value of $R_{S}$ [46] and calculate the average moduli separately for each phase. The results are also plotted in Fig. 13. The jump between the two branches becomes clearer in this plot. Hence, despite the discontinuities of the elastic moduli, the obtained data regarding the bulk and shear moduli are not inconsistent with the jamming scaling $B \propto P^{0}$ and $G \propto P^{\frac{1}{2}}$ in each jammed phase.

\section{CONCLUSIONS}

In summary, we have numerically studied the structural and mechanical properties of the packings of the binary mixtures of particles with large size dispersity. We have presented strong evidence that the system exhibits the first-order phase transition between two distinct jammed phases: L phase, where only large particles are jammed, and LS phase, where 

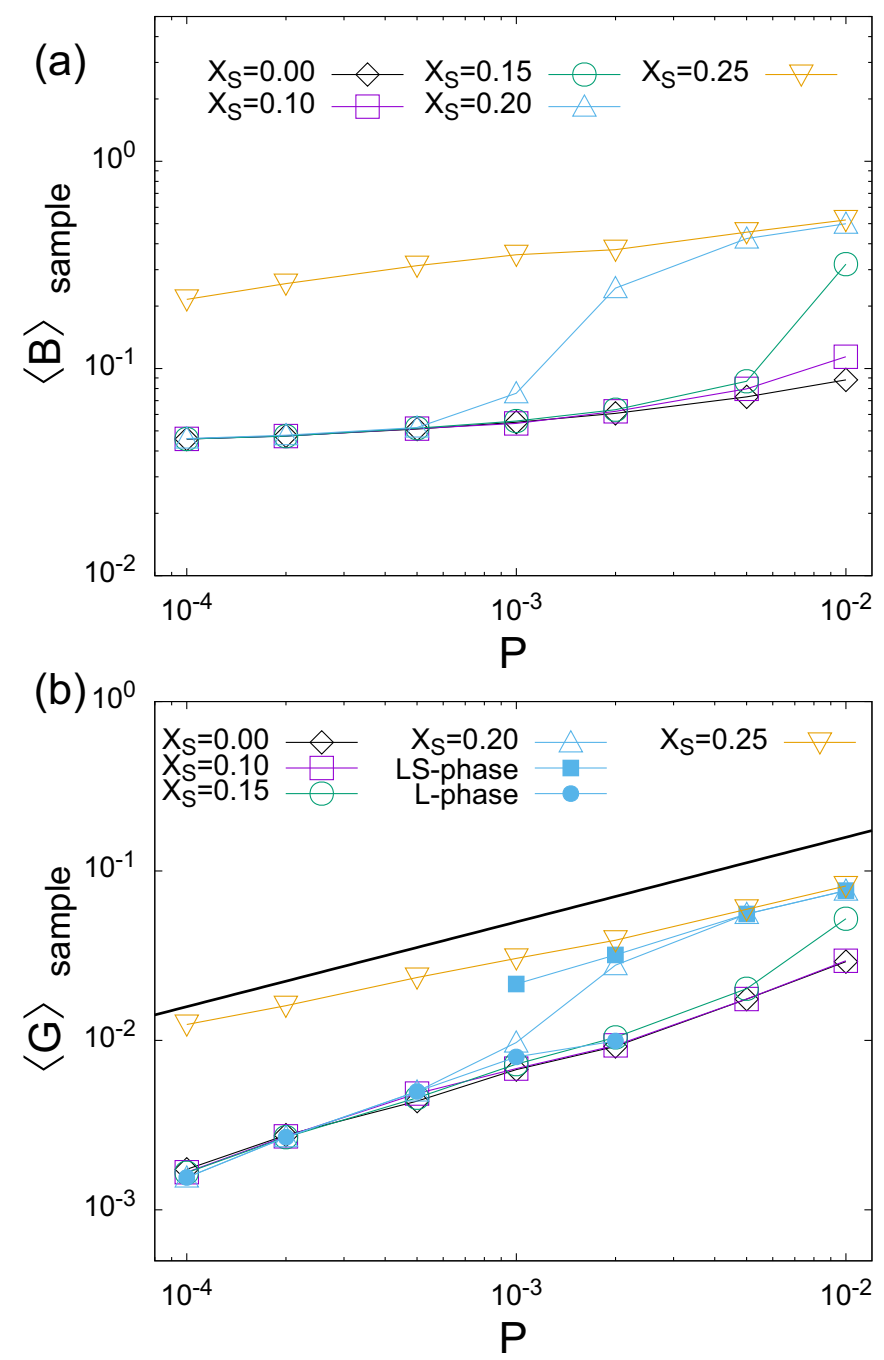

FIG. 13. (a) Bulk modulus and (b) shear modulus plotted as functions of pressure $P$. In (b), the dotted line indicates $P^{1 / 2}$. For $X_{S}=0.2$, the shear modulus averaged separately for the $\mathrm{L}$ phase and the LS phase are also plotted (shown as filled symbols).

both types of particles are jammed. This study was achieved by analyzing the statistics of the fraction $R_{S}$ of small particles that participate in the connecting network. The mean value of $R_{S}$ shows sharp increases at finite $X_{S}$, and the increase becomes progressively steeper when the system size increases. The probability distribution of $R_{S}$ exhibits typical behaviors of the first-order transition. We analyzed the susceptibility $\chi$ defined by the variance of $R_{S}$ and found that it is proportional to the system size. We also analyzed the susceptibility $\tilde{\chi}$ defined by the first derivative of $R_{S}$ and found that it is proportional to the square root of the system size. These results suggest that the present model exhibits a first-order transition whose nature is similar to that in the random-field Ising model.

We have also shown that the elastic moduli can be used as the order parameter of this transition. The LS phase is more rigid than the $\mathrm{L}$ phase. The probability distribution of the bulk and shear moduli behave similar to those of $R_{S}$. Finally, we have shown that, in each phase, our data on these moduli near the jamming transition are not inconsistent with $B \propto P^{0}$ and $G \propto P^{1 / 2}$, the jamming scalings for the monodisperse system.
For nearly monodisperse systems, it has been established that the vibrational and transport properties exhibit the critical behavior near the jamming transition [3,4,6,7,12,14-16]. As we established that the binary mixtures with large size dispersity exhibited another transition, it is interesting to study the vibrational properties of this system.

\section{ACKNOWLEDGMENTS}

We thank K. Hukushima and H. Ikeda for useful discussions. We also thank B. P. Tighe, M. Sperl, and L. E. Silbert for useful information. This work was supported by Japan Society for the Promotion of Science KAKENHI Grants No. JP18H05225, No. JP19H01812, No. JP19K14670, No. JP20H01868, and No. JP20H00128. The computations were partially performed using the Research Center for Computational Science, Okazaki, Japan.

\section{APPENDIX: FORMULATION OF ELASTIC CONSTANTS}

In this Appendix, we introduce the linear response formalism to calculate the elastic constants. We basically follow the formalism of in Ref. [47]. $U$ is a function of both particle positions $\vec{r}_{i}$ and strain tensor $\eta_{\alpha \beta}$. Hereafter, Greek index denotes the spatial index, and Roman index denotes the index of the particles. The stress tensor $t_{\alpha \beta}$ is defined as

$$
\begin{gathered}
t_{\alpha \beta}=\frac{1}{V}\left(\frac{D U}{D \eta_{\alpha \beta}}\right)_{\eta_{\alpha \beta}=0}, \\
=\frac{1}{V}\left(\frac{\partial U}{\partial \eta_{\alpha \beta}}+\sum_{i} \frac{D \vec{r}_{i}}{D \eta_{\alpha \beta}} \cdot \frac{\partial U}{\partial \vec{r}_{i}}\right)_{\eta_{\alpha \beta}=0},
\end{gathered}
$$

where $\frac{D}{D \eta_{\alpha \beta}}$ is the derivatives that impose the mechanical equilibrium $\vec{f}_{i}=-\frac{\partial U}{\partial \vec{r}_{i}}=\overrightarrow{0}$, and the second term vanishes because of this condition. The mechanical equilibrium leads to the following equation

$$
\overrightarrow{0}=\frac{D}{D \eta_{\alpha \beta}}\left(\frac{\partial U}{\partial \vec{r}_{i}}\right)=\frac{\partial^{2} U}{\partial \eta_{\alpha \beta} \partial \vec{r}_{i}}+\sum_{j} \frac{\partial^{2} U}{\partial \vec{r}_{j} \partial \vec{r}_{i}} \frac{D \vec{r}_{j}}{D \eta_{\alpha \beta}},
$$

where $\frac{\partial^{2} U}{\partial \vec{r}_{j} \partial \vec{r}_{i}}=\boldsymbol{H}_{i j}$ is a $3 \times 3$ matrix with $i j$ components of dynamical matrix $\boldsymbol{H}$, and $\frac{\partial^{2} U}{\partial \eta_{\alpha \beta} \partial \vec{r}_{i}}=\overrightarrow{\boldsymbol{\Xi}}_{i, \alpha \beta}$ is the nonaffine force field.

Elastic constants are defined as the second-order derivatives of the energy by the strain tensor $\eta_{\alpha \beta}$ and have following form

$$
C_{\alpha \beta \kappa \chi}=\frac{1}{V}\left(\frac{D^{2} U}{D \eta_{\alpha \beta} D \eta_{\kappa \chi}}\right)_{\eta_{\alpha \beta}=0} .
$$

This is decomposed into two terms as follows

$$
C_{\alpha \beta \kappa \chi}=\frac{1}{V}\left(\frac{\partial^{2} U}{\partial \eta_{\alpha \beta} \partial \eta_{\kappa \chi}}+\sum_{i} \frac{D \vec{r}_{i}}{D \eta_{\alpha \beta}} \cdot \frac{\partial^{2} U}{\partial \vec{r}_{i} \partial \eta_{\kappa \chi}}\right)_{\eta_{\alpha \beta}=0} .
$$

Unlike the stress tensor, the second term is nonzero under mechanical equilibrium and is called the nonaffine correction 
to the elastic constants. The derivatives under constraint is replaced by the nonaffine force field $\vec{\Xi}_{i, \alpha \beta}$, which leads to

$$
C_{\alpha \beta \kappa \chi}=\frac{1}{V}\left(\frac{\partial^{2} U}{\partial \eta_{\alpha \beta} \partial \eta_{\kappa \chi}}-\sum_{i j} \vec{\Xi}_{i, \alpha \beta} \boldsymbol{H}_{i j}^{-1} \vec{\Xi}_{j, \kappa \chi}\right)_{\eta_{\alpha \beta}=0} .
$$

The second term is rewritten by diagonalizing dynamical matrix $\boldsymbol{H}$

$$
\sum_{i j} \vec{\Xi}_{i, \alpha \beta} \boldsymbol{H}_{i j}^{-1} \vec{\Xi}_{j, \kappa \chi}=\sum_{n} \frac{\Xi_{\alpha \beta}^{n} \Xi_{\kappa \chi}^{n}}{\lambda^{n}} .
$$

Here, the nth eigenvalues and eigen vectors of $\boldsymbol{H}$ are represented as $\lambda^{n}$ and $\vec{\Psi}^{n}$, and the inner product of $\vec{\Xi}_{\alpha \beta}$ and
$\vec{\Psi}^{n}$ is $\Xi_{\alpha \beta}^{n}$.

$$
C_{\alpha \beta \kappa \chi}=\frac{1}{V}\left(\frac{\partial^{2} U}{\partial \eta_{\alpha \beta} \partial \eta_{\kappa \chi}}-\sum_{n} \frac{\Xi_{\alpha \beta}^{n} \Xi_{\kappa \chi}^{n}}{\lambda^{n}}\right) .
$$

By evaluating elastic constants $C_{\alpha \beta \kappa \chi}$, bulk moduli $B$ and shear moduli $G$ are calculated as follows,

$$
B=\frac{1}{9}\left(C_{x x x x}+C_{y y y y}+C_{z z z z}+2 C_{x x y y}+2 C_{y y z z}+2 C_{z z y y}\right),
$$

$$
G=\frac{1}{3}\left(C_{x y x y}+C_{y z y z}+C_{z x z x}\right) .
$$

[1] M. Van Hecke, Jamming of soft particles: Geometry, mechanics, scaling and isostaticity, J. Phys.: Condens. Matter 22, 033101 (2010).

[2] C. S. O’Hern, L. E. Silbert, A. J. Liu, and S. R. Nagel, Jamming at zero temperature and zero applied stress: The epitome of disorder, Phys. Rev. E 68, 011306 (2003).

[3] L. E. Silbert, A. J. Liu, and S. R. Nagel, Vibrations and Diverging Length Scales Near the Unjamming Transition, Phys. Rev. Lett. 95, 098301 (2005).

[4] M. Wyart, L. E. Silbert, S. R. Nagel, and T. A. Witten, Effects of compression on the vibrational modes of marginally jammed solids, Phys. Rev. E 72, 051306 (2005).

[5] W. G. Ellenbroek, M. Van Hecke, and W. Van Saarloos, Jammed frictionless disks: Connecting local and global response, Phys. Rev. E 80, 061307 (2009).

[6] M. Wyart, Scaling of phononic transport with connectivity in amorphous solids, Europhys. Lett. 89, 64001 (2010).

[7] V. Vitelli, N. Xu, M. Wyart, A. J. Liu, and S. R. Nagel, Heat transport in model jammed solids, Phys. Rev. E 81, 021301 (2010).

[8] A. Zaccone and E. Scossa-Romano, Approximate analytical description of the nonaffine response of amorphous solids, Phys. Rev. B 83, 184205 (2011).

[9] C. P. Goodrich, A. J. Liu, and S. R. Nagel, Finite-size Scaling at the Jamming Transition, Phys. Rev. Lett. 109, 095704 (2012).

[10] C. P. Goodrich, S. Dagois-Bohy, B. P. Tighe, M. Van Hecke, A. J. Liu, and S. R. Nagel, Jamming in finite systems: Stability, anisotropy, fluctuations, and scaling, Phys. Rev. E 90, 022138 (2014).

[11] E. Lerner, E. Degiuli, G. Düring, and M. Wyart, Breakdown of continuum elasticity in amorphous solids, Soft Matter 10, 5085 (2014).

[12] E. DeGiuli, E. Lernera, C. Britoc, and M. Wyart, Force distribution affects vibrational properties in hard-sphere glasses, Proc. Nat. Acad. Sci. USA 111, 17054 (2014).

[13] K. Karimi and C. E. Maloney, Elasticity of frictionless particles near jamming, Phys. Rev. E 92, 022208 (2015).

[14] H. Mizuno, H. Shiba, and A. Ikeda, Continuum limit of the vibrational properties of amorphous solids, Proc. Natl. Acad. Sci. USA 114, E9767 (2017).
[15] M. Shimada, H. Mizuno, M. Wyart, and A. Ikeda, Spatial structure of quasilocalized vibrations in nearly jammed amorphous solids, Phys. Rev. E 98, 060901(R) (2018).

[16] H. Mizuno and A. Ikeda, Phonon transport and vibrational excitations in amorphous solids, Phys. Rev. E 98, 062612 (2018).

[17] C. F. Schreck, N. Xu, and C. S. O'Hern, A comparison of jamming behavior in systems composed of dimer- and ellipse-shaped particles, Soft Matter 6, 2960 (2010).

[18] K. Shiraishi, H. Mizuno, and A. Ikeda, Vibrational properties of two-dimensional dimer packings near the jamming transition, Phys. Rev. E 100, 012606 (2019).

[19] K. Shiraishi, H. Mizuno, and A. Ikeda, Mechanical and vibrational properties of three-dimensional dimer packings near the jamming transition, J. Phys. Soc. Jpn. 89, 074603 (2020).

[20] X. Huang, Y. Kakuda, and W. Cui, Hydrocolloids in emulsions: Particle size distribution and interfacial activity, Food Hydrocolloids 15, 533 (2001).

[21] L. Zeng, Y. Zhang, C. Bukirwa, W. Li, and Y. Yang, Study of mean diameter and drop size distribution of emulsion drops in a modified rotating disc contactor for an emulsion liquid membrane system, RSC Adv. 5, 89959 (2015).

[22] S. Kwok, R. Botet, L. Sharpnack, and B. Cabane, Apollonian packing in polydisperse emulsions, Soft Matter 16, 2426, (2020).

[23] S. R. Derkach, Rheology of emulsions, Adv. Colloid Interface Sci. 151, 1 (2009).

[24] J. Clara-Rahola, T. A. Brzinski, D. Semwogerere, K. Feitosa, J. C. Crocker, J. Sato, V. Breedveld, and Eric R. Weeks, Affine and nonaffine motions in sheared polydisperse emulsions, Phys. Rev. E 91, 010301 (2015).

[25] W. C. K. Poon, The physics of a model colloidpolymer mixture, J. Phys.: Condens. Matter 14, R859 (2002).

[26] F. Sciortino and P. Tartaglia, Glassy colloidal systems, Adv. Phys. 54, 471 (2005).

[27] T. Sentjabrskaja, E. Babaliari, J. Hendricks, M. Laurati, G. Petekidis, and S. U. Egelhaaf, Yielding of binary colloidal glasses, Soft Matter 9, 4524 (2013). 
[28] T. Sentjabrskaja, A. R. Jacob, S. U. Egelhaaf, G. Petekidis, T. Voigtmann, and M. Laurati, Binary colloidal glasses: Linear viscoelasticity and its link to the microscopic structure and dynamics, Soft Matter 15, 2232 (2019).

[29] S. Yerazunis, S. W. Cornell, and B. Wintner, Dense random packing of binary mixtures of spheres, Nature (London) 207, 835 (1965)

[30] J. Zheng, W. B. Carlson, and J. S. Reed, The packing density of binary powder mixtures, J. Eur. Ceram. Soc. 15, 479 (1995).

[31] R. S. Farr and R. D. Groot, Close packing density of polydisperse hard spheres, J. Chem. Phys. 131, 244104 (2009).

[32] L. Meng, P. Lu, and S. Li, Packing properties of binary mixtures in disordered sphere systems, Particuology 16, 155 (2014).

[33] D. J. Koeze, D. Vågberg, B. B. T. Tjoa, and B. P. Tighe, Mapping the jamming transition of bidisperse mixtures, Europhys. Lett. 113, 54001 (2016).

[34] I. Prasad, C. Santangelo, and G. Grason, Subjamming transition in binary sphere mixtures, Phys. Rev. E 96, 052905 (2017).

[35] S. Pillitteri, G. Lumay, E. Opsomer, and N. Vandewalle, From jamming to fast compaction dynamics in granular binary mixtures, Sci. Rep. 9, 7281 (2019).

[36] N. Xu and E. S. C. Ching, Effects of particle-size ratio on jamming of binary mixtures at zero temperature, Soft Matter 6, 2944 (2010).

[37] N. Kumar, V. Magnanimo, M. Ramaioli, and S. Luding, Tuning the bulk properties of bidisperse granular mixtures by small amount of fines, Powder Technol. 293, 94 (2016).

[38] J. C. Petit, N. Kumar, S. Luding, and M. Sperl, Additional Transition Line in Jammed Asymmetric Bidisperse Granular Packings, Phys. Rev. Lett. 125, 215501 (2020).

[39] E. Bitzek, P. Koskinen, F. Gähler, M. Moseler, and P. Gumbsch, Structural Relaxation Made Simple, Phys. Rev. Lett. 97, 170201 (2006).
[40] W. Lechner and C. Dellago, Accurate determination of crystal structures based on averaged local bond order parameters, J. Chem. Phys. 129, 114707 (2008).

[41] R. L. C. Vink, K. Binder, and H. Löwen, Colloid-polymer mixtures in random porous media: Finite size scaling and connected versus disconnected susceptibilities, J. Phys.: Condens. Matter, 20, 404222 (2008).

[42] M. Ozawa, L. Berthier, G. Biroli, A. Rosso, and G. Tarjus, Random critical point separates brittle and ductile yielding transitions in amorphous materials, Proc. Nat. Acad. Sci. USA 115, 6656 (2018).

[43] In practice, we numerically evaluate $\tilde{\chi}$ by $\tilde{\chi}\left(X_{S}\right)=$ $\frac{1}{2 \Delta X_{S}}\left(\left\langle R_{S}\right\rangle_{\text {sample }}\left(X_{S}+\Delta X_{S}\right)-\left\langle R_{S}\right\rangle_{\text {sample }}\left(X_{S}-\Delta X_{S}\right)\right), \quad$ where $\Delta X_{S}$ is the discretization width of $X_{S}$. Typically, $\Delta X_{S} \approx 0.004$ for $N_{S}=4000$ and $8000, \Delta X_{S} \approx 0.002$ for $N_{S}=12000$, and $\Delta X_{S} \approx 0.001$ for $N_{S}=16000$, which correspond to the insertion of two large particles. We check that the results do not change much even if only the first or second half of samples is used for the analysis.

[44] In practice, $X_{S}\left(\left\langle R_{S}\right\rangle_{\text {sample }}=0.7\right)$ and $X_{S}\left(\left\langle R_{S}\right\rangle_{\text {sample }}=0.3\right)$ are evaluated using the linear interpolation of $\left\langle R_{S}\right\rangle_{\text {sample }}\left(X_{S}\right)$ presented in Fig. 1.

[45] K. Binder and D. P. Landau, Finite-size scaling at first-order phase transitions, Phys. Rev. B 30, 1477 (1984).

[46] A packing is judged to be in the $\mathrm{L}$ phase when $R_{S}<0.5$ and in the LS phase when $R_{S} \geqslant 0.5$.

[47] A. Lemaître and C. Maloney, Sum rules for the quasi-static and visco-elastic response of disordered solids at zero temperature, J. Stat. Phys. 123, 415 (2006).

Correction: The given name of the last author contained an error and has been fixed. 